\title{
A chromosomal hotspot for multiple rearrangements associated with genetic instability of Streptomyces ambofaciens DSM 40697
}

\author{
Philippe Demuyter, $\dagger$ Dominique Schneider, Pierre Leblond, Jean-Marc Simonet and \\ BERNARD DECARIS*
}

Laboratoire de Génétique et Microbiologie, Faculté des Sciences de l'Université de Nancy I, BP 239, Institut de Biotechnologies, 54506 Vandoeuvre-lès-Nancy, France

(Received 18 September 1990; revised 12 November 1990; accepted 19 November 1990)

\begin{abstract}
Genetic instability in Streptomyces ambofaciens DSM 40697 involves genomic rearrangements such as amplifications and deletions of particular DNA sequences. Most amplifications were located in two amplifiable regions, one of which, called AUD6 (amplifiable unit of DNA no. 6) was revealed to be a rearrangement hotspot. Indeed, 30\% of the mutant strains studied had amplifications, deletions or both at the AUD6 locus. This locus contains several reiterations which are specific to this AUD. Moreover, one of the endpoints of the AUD6 shows homology with an internal sequence. Deletions occurred exclusively at one side of the amplified DNA sequence (ADS) and removed part of the proximal copy of this ADS, leading to the conclusion that multiple rearrangements can occur at this AUD locus.
\end{abstract}

\section{Introduction}

Streptomycetes often exhibit a high degree of genetic instability and many characters have been reported to be unstable (for reviews, see Hütter \& Eckhardt, 1988; Leblond et al., 1990a; Birch et al., 1990). In Streptomyces ambofaciens DSM 40697, a basic genetic instability produces pigment-defective $\left(\mathrm{Pig}^{-}\right)$colonies at a frequency of about $1 \%$. A second aspect of this genetic instability, called hypervariability, has been characterized: $87 \%$ of the Pig $^{-}$colonies generated by the basic genetic instability lead to a phenotypically heterogeneous progeny without a preponderant phenotype (Leblond et al., 1989). All the strains studied here, which were isolated among a phenotypically heterogeneous progeny, were called 'hypervariability-derived' strains.

Genetic instability could be due to plasmid loss (Usdin et al., 1985) or chromosomal rearrangements by formation of large deletions including the unstable gene (for a review, see Hütter \& Eckhardt, 1988).

Highly amplified DNA sequences (ADSs) have been detected within the DNA isolated from mutants of many

† Present address: Institut de Biologie Moléculaire et Cellulaire, Laboratoire de Génétique, 15 rue Descartes, 67084 Strasbourg, France.

Abbreviations: ADS, amplified DNA sequence; AUD, amplifiable unit of DNA; IHS, internal homologous sequence; $\mathrm{Pig}^{-}$, pigmentdefective; WT, wild-type.
Streptomyces species (for a review, see Cullum et al., 1986). These amplifications appeared either spontaneously (Schrempf, 1983; Altenbuchner \& Cullum, 1984, 1985) or after various treatments (Robinson et al., 1981; Hasegawa et al., 1985). In S. ambofaciens DSM 40697, several ADSs which were isolated from 'hypervariability-derived' strains have already been described (Demuyter et al., 1988; Leblond et al., 1989). DNA amplifications are frequently associated with large deletions found immediately adjacent or in close proximity to the ADS (Hasegawa et al., 1985; Birch et al., 1989). Altenbuchner \& Cullum $(1984,1985)$ showed that chloramphenicol-sensitive mutant strains of Streptomyces lividans were extremely unstable: in their progeny, spontaneous arginine auxotrophs ( $\left.\mathrm{Arg}^{-}\right)$appeared at a frequency of about $25 \%$. The $\mathrm{Arg}^{-}$strains showed the amplification of a particular $5.7 \mathrm{~kb}$ chromosomal fragment and a deletion at one side of the amplifiable region. In different Streptomyces species, the extent and the localization of chromosomal deletions are quite different. In Streptomyces glaucescens, the deletions were found to extend to over $800 \mathrm{~kb}$ without affecting the growth (Birch et al., 1989). In Streptomyces coelicolor, silent chromosomal arcs exist where very few markers were localized (Chater \& Hopwood, 1984). These arcs might contain large stretches of 'dispensable' DNA. In bacteria other than Streptomyces (for example, Escherichia coli and Bacillus subtilis), it has been shown that the 
frequency of deletion formation on plasmids is increased by the presence of palindromic sequences or direct repeats (DasGupta et al., 1987; Peeters et al., 1988). The same phenomenon could exist at the chromosome level so that the deletion formation could then be explained by intramolecular recombination.

In $S$. ambofaciens DSM 40697, two amplifiable regions have been characterized. One of these regions, the AUD6 (amplifiable unit of DNA no. 6) locus, is described in this work. The lineages of different mutant strains were analysed for the rearrangements found at the AUD6 locus. This region seems to be a rearrangement hotspot. These rearrangements could proceed through several steps.

\section{Methods}

Bacterial strains and plasmid. S. ambofaciens DSM 40697 (Hütter, 1967) was used as the wild-type (WT) strain; it was obtained from the Deutsche Sammlung von Mikroorganismen. Amplified mutant strains spontaneously isolated from the WT strain are listed in Table 1.

E. coli JM109 (Yanisch-Perron et al., 1985) and the plasmid pBluescriptKS (Short et al., 1988) were the host and vector for cloning.

Media and culture conditions. Streptomyces strains were grown at $37^{\circ} \mathrm{C}$ on plates of Hickey Tresner (HT) medium (Pridham et al., $1956 / 57)$ for mutant isolation and spore production. Large-scale DNA isolation was carried out after growth for $48 \mathrm{~h}$ at $37^{\circ} \mathrm{C}$ in YEME liquid medium (Hopwood et al., 1985) supplemented with glycine $(0 \cdot 25 \%)$. Small-scale DNA isolations were performed after growth for $24 \mathrm{~h}$ at $37^{\circ} \mathrm{C}$ in HT liquid medium supplemented with glycine $(0.25 \%)$.

E. coli strains were grown at $37^{\circ} \mathrm{C}$ in LB liquid medium (Maniatis $e t$ al., 1982).

Isolation of total DNA and restriction analyses. DNA extraction and purification were performed according to Demuyter et al. (1988). Restriction enzymes were purchased from Boerhinger Mannheim and used according to the manufacturer's recommendations. DNA fragments were electrophoresed on $0.8 \%$ agarose gels according to Maniatis

Table 1. Strains with amplified DNA used in this work

\begin{tabular}{|c|c|c|c|}
\hline Strain & Origin & $\begin{array}{c}\text { Type of } \\
\text { amplification }\end{array}$ & Deletion* \\
\hline NSA6 & Hypervariable lineage & ADS6 & $\Delta 2$ \\
\hline NSA60 & Hypervariable lineage & ADS60 & ND \\
\hline NSA101 & Hypervariable lineage & ADS101 & $\Delta 2$ \\
\hline NSA 102 & Hypervariable lineage & ADS 102 & + \\
\hline NSA631 & Derived from NSA6 & ADS6 & ND \\
\hline NSA641 & Derived from NSA6 & ADS6 & ND \\
\hline $\begin{array}{l}\text { NSA641 }_{1}, \\
\text { NSA641 }_{2}\end{array}$ & Derived from NSA641 & ADS6 & ND \\
\hline
\end{tabular}

* $\Delta 2$ represents a deletion which removes a part of the proximal copy of the corresponding ADS without affecting the $12 \mathrm{~kb} \mathrm{BamHI}$ fragment (Fig. 2). For strain NSA102, + indicates the presence of a deletion at the left side of the ADS, but we were not able to localize the right endpoint of this deletion precisely.

ND, Not determined. et al. (1982). Bacteriophage $\lambda$ DNA digested with HindIII was used as size standard (Daniels et al., 1983).

Cloning of DNA fragments. The $2.9 \mathrm{~kb}$ BamHI joint-segment generated by tandem reiteration of AUD6 was isolated from total DNA of NSA6. This fragment was cloned and used as probe S1 (Fig. 1). The left part of $S 1$ in the WT AUD6 was called $S I_{L}$ and the right part $S 1_{R}$. The 3.1 kb BamHI left flanking fragment of AUD6 was cloned from total DNA of NSA60 and used as probe S2.

The probes $\mathrm{S} 3$ and $\mathrm{S} 4$, which correspond to the $3.5 \mathrm{~kb}$ and $1.9 \mathrm{~kb}$ BamHI fragments, respectively, of AUD6, were recovered by elution from the total DNA of strain NSA6. To verify that these two probes were not contaminated with fragments of the same size, controls similar to those described by Demuyter et al. (1988) were done.

${ }^{32} \mathrm{P}$ labelling of DNA, Southern blotting and hybridizations. DNA probes were ${ }^{32}$ P-labelled (Amersham) according to Feinberg \& Vogelstein (1983) using the Multiprime DNA labelling system kit (Amersham) or a nick-translation kit (Amersham). The labelling was carried out either with purified DNA or directly in the agarose matrix.

For hybridization experiments, Southern blottings (Southern, 1975) were done by the capillary transfer method with the Vacugene system (LKB) onto Hybond-N membranes (Amersham). The DNA was depurinated with $0.25 \mathrm{M}-\mathrm{HCl}$ for 30 min before denaturation (in $1.5 \mathrm{M}$ $\mathrm{NaCl}, 0.5 \mathrm{M}-\mathrm{NaOH}$ for $1 \mathrm{~h}$ ) and neutralization (in $1.5 \mathrm{M}-\mathrm{Tris} / \mathrm{HCl}$, $1.5 \mathrm{M}-\mathrm{NaCl}$ for $30 \mathrm{~min})$, and vacuum-transferred with $20 \times \operatorname{SSC}(1 \times$ SSC is $0.15 \mathrm{M}$-sodium chloride, $0.015 \mathrm{M}$-sodium citrate) for $90 \mathrm{~min}$. Prehybridization, hybridization and washing conditions were as described by Demuyter et al. (1988).

\section{Results}

\section{Structure of the AUD6 region}

Restriction patterns of amplified DNA extracted from mutant strains isolated from hypervariable lineages allowed us to map eight new overlapping amplifiable sequences with respect to the three AUDs previously described by Demuyter et al. (1988) (Fig. 1). Two of these AUDs, AUD102 and AUD420, were indistinguishable by the methods used (restriction analyses and hybridization experiments). Apart from this case, all the sequences were different in length. A twelfth amplification (ADS7) was recovered, but it may not be independent of the others since it was derived from the ADS6-containing strain. Nothing in the map revealed the presence of long direct repeats. This was confirmed by hybridization for ADS6. This family of amplifications defines the AUD6 region.

In order to detect rearrangements other than amplifications of the AUD6 locus in various mutant strains isolated from stable and hypervariable lineages, Southern blots of BamHI digests of total DNA from the WT and strain NSA6 were hybridized with the S1 probe. The hybridization pattern of WT DNA (Fig. $2 a$, lane 1) revealed three bands: $15 \mathrm{~kb}, 12 \mathrm{~kb}$ and $3 \cdot 1 \mathrm{~kb}$. According to the restriction map of the AUD6 region, the $15 \mathrm{~kb}$ and $3 \cdot 1 \mathrm{~kb}$ fragments were the flanking sequences of AUD6. S1 was used on 31 restriction patterns of independently 


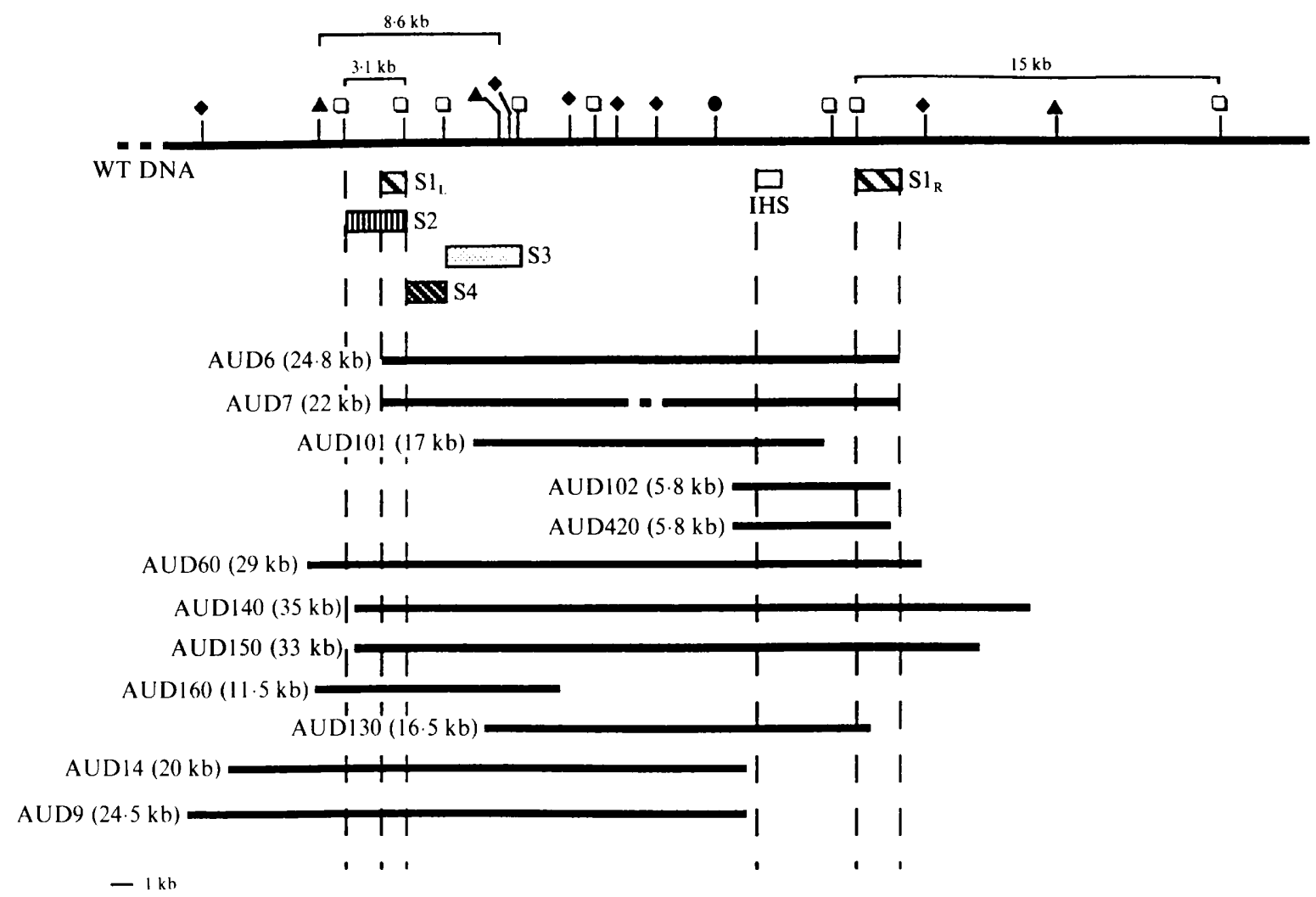

Fig. 1. Map of the AUD6 locus of S. ambofaciens DSM 40697. The eleven independent AUDs belonging to the AUD6 family are shown. AUD7 contains an internal deletion of the WT AUD (symbolized by dotted lines) and is derived from strain NSA6. The following restriction enzymes were used : $B a m H I(\square), P v u I I(\diamond)$ and $X h o I(\boldsymbol{\Lambda})$. The following symbols are used for the probe fragments: $\mathbf{\nabla \nabla}, \mathrm{SI}$; 孟而, S2; $\square$, IHS, $\square$. S3; MI, S4. S1 is divided into $S 1_{R}$ and $S 1_{L}$ for the right and the left part of S1, respectively. IHS is a DNA region which shows homology to $S 1_{R}$. The vertical dotted lines show alignment with respect to probe fragments.

(a)

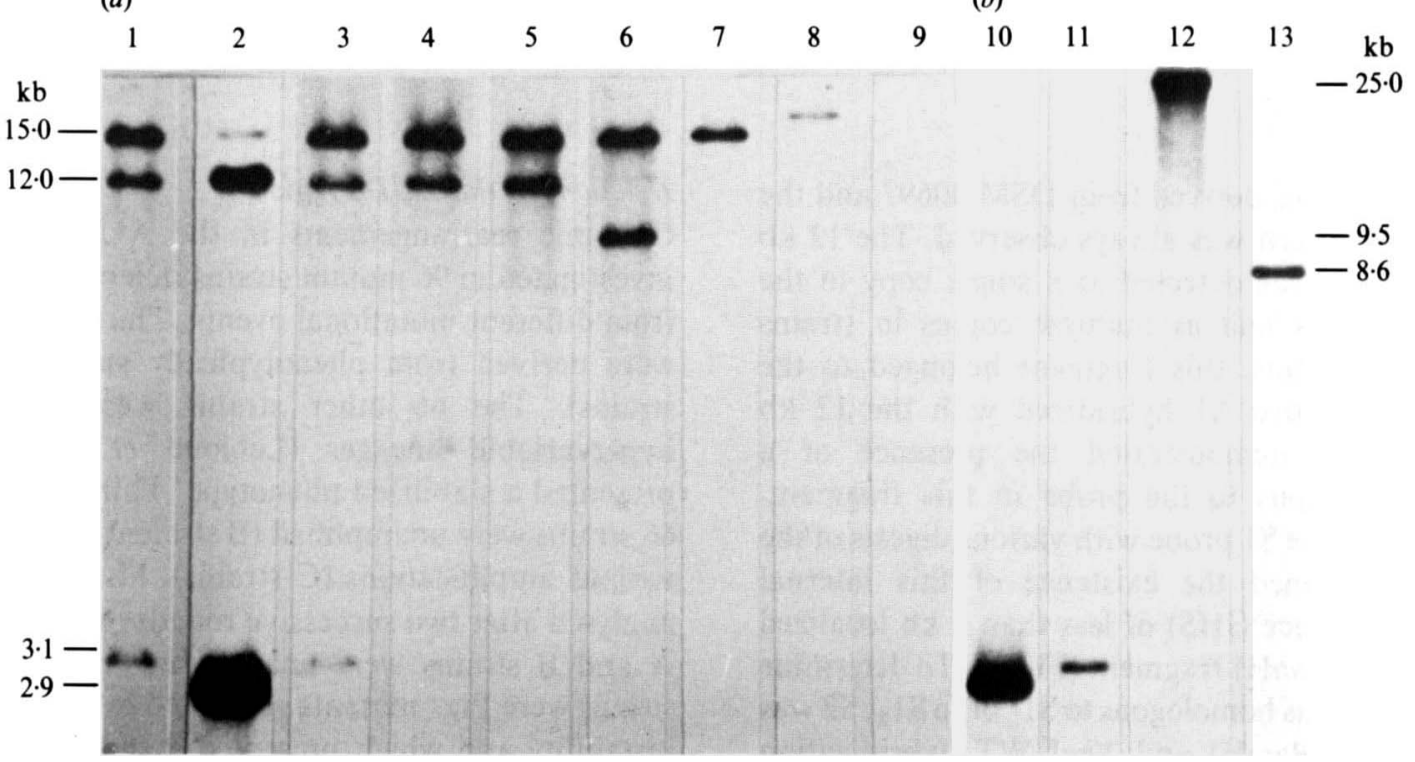

Fig. 2. (a) Hybridization patterns of the total DNA of the WT strain and of various mutant strains digested with BamHI. The probe used was the cloned endpoints of the ADS6 (S1). Lane 1, WT; lane 2, amplified strain NSA6; lane 3, pattern of the strains without detectable rearrangement in the AUD6 region ( $\Delta 0)$; lanes 4 to 8, patterns which correspond to partial deletions of the AUD6 ( $\Delta 1$ to $\Delta 5)$; lane 9, pattern which corresponds to a total deletion of the AUD6 ( $\Delta 6)$. (b) BamHI (lanes 10 and 11 ) and XhoI (lanes 12 and 13 ) hybridization patterns of the total DNA of the WT strain (lanes 11 and 13) and of strain NSA6 (lanes 10 and 12). The probe used was S2 which was cloned. A greater amount of DNA and a longer exposure for the WT and mutant strains explain why a single-copy signal in the WT and mutant strains (lanes 1, 4, 5, and 6) is more intense than a single-copy signal in NSA6 (fragment of $15 \mathrm{~kb}$, lane 2). 


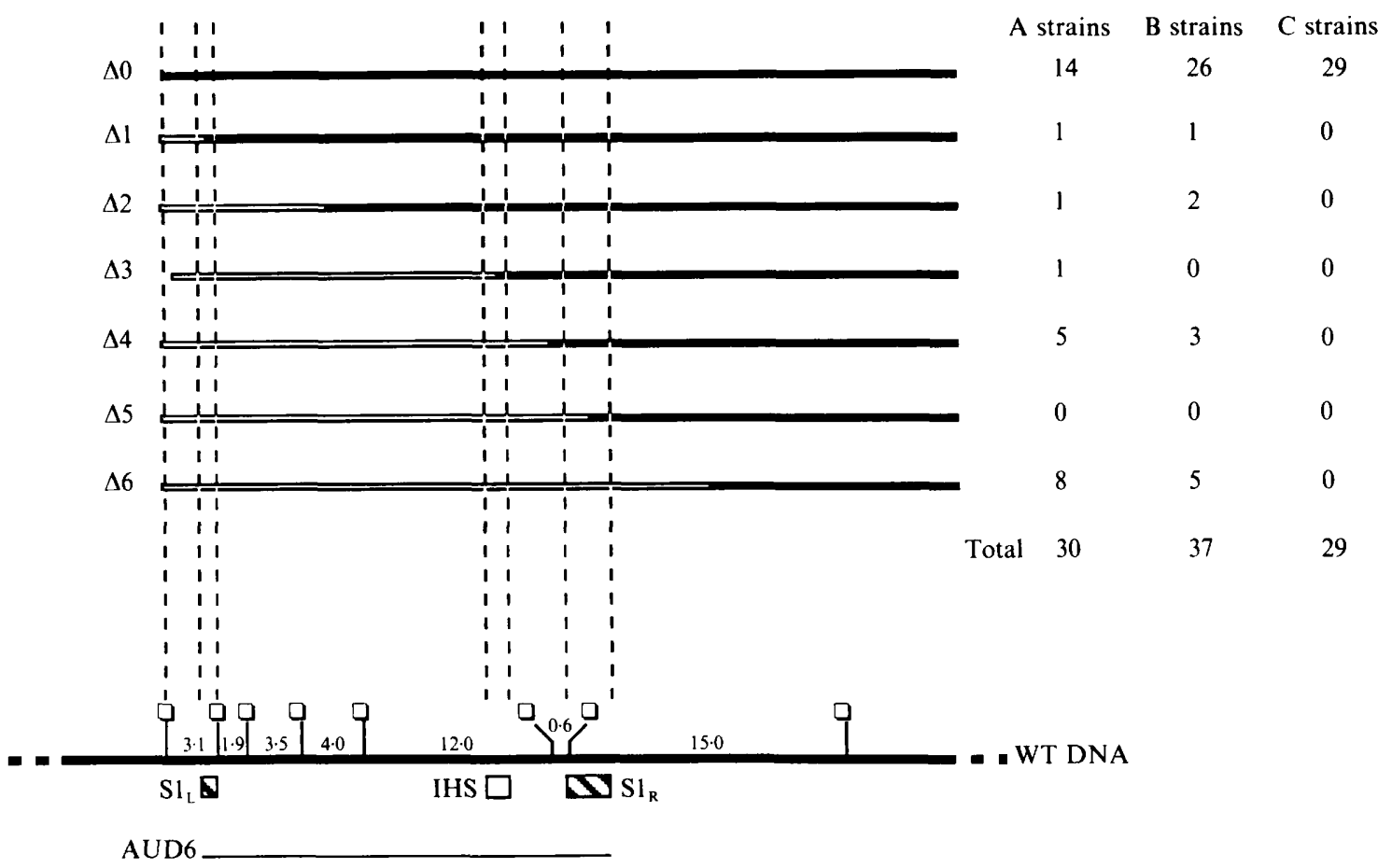

Fig. 3. Rearrangements in the AUD6 region. $\Delta 1$ to $\Delta 6$ are the different observed deletions. The vertical dotted lines delimit areas with respect to the locations which defined the kind of deletion. The black lines over the WT map represent the sequences which are not deleted. The white boxes are the sequences which are deleted in the different mutant strains. The right endpoint of the deletion is localized in each case within a fragment delimited by the vertical dotted lines but the precise endpoint cannot be more accurately localized. For $\Delta 0$, no deletion is detectable. The BamHI restriction sites $(\square)$ are shown. The extent of AUD6 and the location of S1 (D) and IHS $(\square)$ are also indicated. The number of clones showing rearrangements in the AUD6 region for different mutant strains (A, B and C strains) are shown to the right of the figure. The A strains were derived from phenotypically stable lineages. The B strains were isolated from hypervariable lineages, which present a stabilized phenotype and which are not amplified. The $\mathrm{C}$ strains were isolated from hypervariable lineages carrying amplifications. The $29 \mathrm{C}$ strains studied contain ADSs not located in the AUD6 region and were chosen among the 48 amplified strains recovered in the laboratory. The $\Delta 5$ rearrangement is another potential deletion event which was encountered in another experiment.

isolated WT colonies derived from DSM 40697 and the same banding pattern was always observed. The $12 \mathrm{~kb}$ Bam HI fragment was detected as a single copy in the unamplified strains and as multiple copies in strains carrying ADS6. Thus, this fragment belonged to the AUD6. The fact that S1 hybridized with the $12 \mathrm{~kb}$ BamHI fragment demonstrated the presence of a sequence homologous to the probe in this fragment. Hybridization of the S1 probe with various digests of the WT DNA confirmed the existence of this internal homologous sequence (IHS) of less than $1 \mathrm{~kb}$ localized within the $12 \mathrm{~kb} \mathrm{Bam} \mathrm{HI} \mathrm{fragment} \mathrm{(Fig.} \mathrm{1).} \mathrm{To} \mathrm{determine}$ whether the IHS was homologous to $S 1_{L}$ or to $S 1_{R}, S 2$ was used as a probe. BamHI and $\mathrm{XhoI}$ WT hybridization patterns (Fig. $2 b$, lanes 11 and 13 ) revealed only a $3 \cdot 1 \mathrm{~kb}$ BamHI band and a $8.6 \mathrm{~kb}$ XhoI band which both originate from the left side of the AUD6 locus. No signal corresponding to the IHS was detected showing that it was homologous only to the right extremity of the AUD6.

\section{Deletions in the AUD6 region}

Genomic rearrangements in the AUD6 region were investigated in 96 mutant strains independently isolated from different mutational events. Thirty mutant strains were derived from phenotypically stable lineages (A strains). The 66 other strains were isolated from hypervariable lineages (Leblond et al., 1989) and presented a stabilized phenotype. Thirty-seven of these 66 strains were unamplified (B strains) and 29 presented various amplifications (C strains). Mutant clones were analysed after two successive rounds of subcloning. The A and B strains were unamplified mutant strains. A strains were $\mathrm{Pig}^{-}$mutants generated by the basic genetic instability and which presented a stable phenotype. B strains derived from the $87 \%$ of $\mathrm{Pig}^{-}$colonies which presented a phenotypic heterogeneity in their progeny (hypervariable lineages) (Leblond et al., 1989). C strains derived from hypervariable lineages and are amplified strains (carrying amplifications localized in a locus distinct from AUD6). 


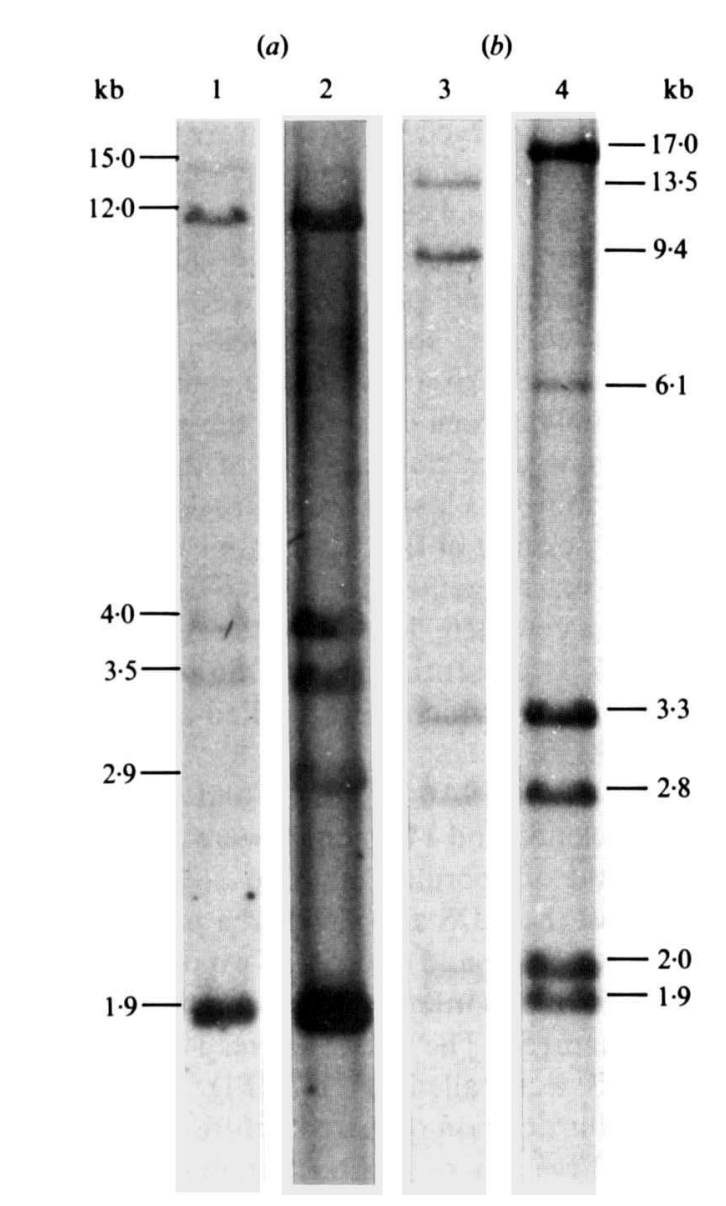

Fig. 4. Hybridization patterns using the $S 4$ probe which is the $1.9 \mathrm{~kb}$ BamHI fragment of ADS6. The total DNA of the WT strain (lanes 1 and 3) and of strain NSA6 (lanes 2 and 4) was digested with BamHI (a) and PvuII $(b)$. The BamHI patterns revealed a strong hybridization with a $1.9 \mathrm{~kb}$ fragment (which corresponds to the probe) and with four other fragments whose sizes corresponded to those of the AUD6 region. The same result was obtained with the PvuII patterns.

Southern blots of BamHI digests of total DNA from mutant clones were probed with the $\mathrm{S} 1$ probe. The majority of the unamplified mutant strains $[40 /(30 \mathrm{~A}$ strains +37 B strains)] (Fig. 3) were unaltered in the AUD6 region (Fig. 2a, lane 3). Six different hybridization patterns were revealed in $16 / 30 \mathrm{~A}$ strains and in 11/37 B strains (Fig. 3). A seventh potential pattern may exist (called $\Delta 5$ ) but was found in another experiment described in the last section of Results. Some or all of the bands of the characteristic WT BamHI pattern $(15 \mathrm{~kb}, 12$ $\mathrm{kb}, 3 \cdot 1 \mathrm{~kb}$ ) were missing in altered patterns (Fig. $2 a$ ). These differences can be explained by partial ( $\Delta 1$ to $\Delta 5)$ or complete $(\Delta 6)$ deletions of the AUD6. A partial deletion of the AUD6 generated a fragment which can hybridize with $\mathrm{S} 1$ according to the localization of the right endpoint of the deletion (Fig. 2a, lanes 4 and 6).
This endpoint could also be located between the $3 \cdot 1 \mathrm{~kb}$ and $12 \mathrm{~kb}$ length fragments $(\Delta 2)$ or inside the $12 \mathrm{~kb}$ fragment between the IHS and the $0 \cdot 6 \mathrm{~kb}$ sequences $(\Delta 4)$ (Fig. 2a, lanes 5 and 7).

All the left-hand deletion endpoints were outside the AUD6 region and therefore we assumed that a deletion polarity exists. For example, no strain was deleted for $\mathrm{S} 1_{\mathrm{R}}$ without deletion of the $12 \mathrm{~kb} \mathrm{BamHI}$ fragment. These observations could result from a selection imposed by the presence of an essential gene to the right of the AUD. The deletion of the entire AUD $(\Delta 6)$ would not affect this essential gene. Nevertheless, the deletions could be initiated through particular structures or sequences lying within the left region of the AUD. The frequencies of different classes of rearrangements were not significantly different in the stable clones (A strains) and in the unamplified hypervariable clones (B strains) (Fig. 3).

Hybridization patterns of 29 different strains carrying amplifications not originating from the AUD6 region revealed no rearrangement in the AUD6 locus.

The rearrangements were also studied in three strains carrying amplifications localized in the AUD6 locus: ADSs 6, 101 and 102. In the ADS6 DNA, two faint bands of $15 \mathrm{~kb}$ and $3 \cdot 1 \mathrm{~kb}$ and two heavy bands of $12 \mathrm{~kb}$ and $2.9 \mathrm{~kb}$ were the expected signals in a BamHI hybridization pattern (Fig. 1). However, the $3 \cdot 1 \mathrm{~kb}$ fragment of the left flanking sequence of the ADS6 was absent or masked by the heavy spot of the $2.9 \mathrm{~kb}$ reiterated fragment (Fig. $2 a$, lane 2). The flanking sequence was investigated in NSA6 using S2 as a probe (Fig. 1) and hybridized with Southern blots of BamHI and $X$ hoI digests of total DNA from strain NSA6 (Fig. $2 b$, lanes 10 and 12 ). The expected band corresponding to the same flanking sequence, which is a $8.6 \mathrm{~kb} X h o \mathrm{I}$ fragment, disappeared in the XhoI pattern of the NSA6 strain. Moreover, no band other than the $25 \mathrm{~kb}$ fragment, which corresponded to the ADS, was present. The same result was observed for the BamHI pattern of the NSA6 strain, which presented only the $2.9 \mathrm{~kb}$ reiterated fragment. The amplification of ADS6 was associated with a deletion which could be localized at the left side of the AUD and which could remove a part of the proximal copy of the ADS, since no joint-fragment was detected in the hybridization patterns after deletion.

A deletion could also have taken place at the left side of the ADS101 and ADS102 since no signal was obtained with Southern blots of BamHI and XhoI digests of total DNA from strains NSA101 and NSA102 probed with S2 (data not shown).

In order to specify the right endpoints of the deletions associated with the amplification in the three strains NSA6, NSA101 and NSA102, the four probes S1, S2, S3 and $\mathrm{S} 4$ were used. 
Hybridizations were carried out using $\mathrm{S} 4$ and the total DNA of NSA6 digested with BamHI and PvuII (Fig. 1). In the WT strain, S4 hybridized with five BamHI fragments (Fig. 4, lane 1) whose sizes corresponded to those of the AUD6 region. Similar results were obtained with PvuII (Fig. 4, lane 3). Thus, S4 showed several homologous sequences in the AUD6. Since no other band was present, these homologies were not dispersed on the genome but were specific to this locus. This probe is therefore a useful tool for studying the rearrangements in amplified strains. The different intensities of the signals in Fig. 4 could be explained if the homologies of $\mathrm{S} 4$ were not perfect in all the fragments.

The BamHI and PvuII hybridization patterns of the total DNA of NSA6 probed with S4 confirmed the homologies found in the WT (Fig. 4, lanes 2 and 4). The Bam HI pattern revealed an additional $2.9 \mathrm{~kb}$ band. Since this fragment cannot correspond to S1 (because S1 did not hybridize with S4), it might be a new fragment generated by the deletion. The PvuII pattern showed a $6.1 \mathrm{~kb}$ fragment which could constitute the new fragment in question. These results suggested that the deletion, at the left side of the ADS6, removed part of the proximal copy of the ADS and that the right endpoint of this deletion was localized in the $1.9 \mathrm{~kb}$ BamHI fragment.

The same experiments were carried out with NSA101 and NSA 102 using S3 and S4 as probes (data not shown). This led to the conclusion that in NSA101, a deletion occurred at the left side of the ADS 101 and removed part of the proximal copy of the ADS. Moreover, hybridizations with S3 confirmed the homologies found with S4 (data not shown). Hybridizations with the DNA of strain NSA 102 strain showed that the fragments corresponding to $\mathrm{S} 3$ and $\mathrm{S} 4$ were deleted in this strain (data not shown) and since this ADS was shorter than the other two, we were unable to investigate whether the deletion removed part of the proximal copy of the ADS102.

The fact that the proximal copy of an ADS was partially deleted implies that a deletion event occurred after the amplification process (or at least after a first step of duplication to preserve one intact copy of the AUD). This process could remove several copies of the ADS. The deletion events presented the same polarity in the amplified strains as in the unamplified strains.

\section{Instability of the ADS6 amplification}

In order to test whether an ADS was lost by successive steps of deletion, the stability of the amplification was studied by hybridization experiments with $\mathrm{S} 1$ in several mutant clones belonging to the NSA6 lineage. Four successive rounds of sporulation were carried out from the amplified progenitor NSA6 and the different studied clones were randomly isolated as single colonies in this lineage for the first two rounds of sporulation. Only colonies which conserved the ADS were subcloned for the third round of sporulation and only the colonies with a very low copy number of the amplification were subcloned for the fourth round of sporulation.

The NSA6 amplified progenitor led, after the first round of sporulation, to $5 / 5$ studied clones which conserved the ADS. After the second round of sporulation, 12 randomly chosen colonies in the progeny of the NSA6 progenitor were studied: four of them presented the ADS, five showed a loss of the entire ADS associated with a partial deletion of the AUD6 (one with a $\Delta 2$ and four with a $\Delta 3$ rearrangement) and three had also lost the ADS but with a complete deletion of the AUD $(\Delta 6)$. One colony (NSA631) presenting the ADS6 was subcloned and the eight clones which were studied in the progeny retained the ADS.

Another colony (NSA641) which had presented the ADS was subcloned and 14 colonies were obtained after the third round of sporulation. Five out of 14 clones studied had lost the ADS and also had a partial deletion of the AUD (three $\Delta 2$ and two $\triangle 3$ ). Two out of 14 had a modified ADS with an internal deletion removing part of the $12 \mathrm{~kb}$ fragment. The AUD corresponding to this modified ADS was called AUD7 (Fig. 1). This fact implied that this deletion occurred before the amplification process. Five out of fourteen showed the typical ADS6 pattern and 2/14 studied clones (NSA641 ${ }_{1}$ and NSA641 $1_{2}$ ) presented the amplified pattern but the hybridization signals were weaker and the amplification was undetectable on ethidium bromide stained agarose gels. These two strains were then subcloned and in all the clones tested (58), the ADS6 was lost in association with a partial or complete deletion of the AUD6. The weaker hybridization signals of strains NSA641 ${ }_{1}$ and NSA641 could correspond to a transitory step of amplification loss and the next step would be the total loss without return to an intact AUD.

Thus, the ADS6 was an unstable amplification and when this amplification was lost, the AUD6 was never intact and $\Delta 2$ seemed to be the most frequent deletion observed (60/96 studied clones). There were also $\Delta 3$ and $\Delta 6$ clones. In one case, a modified amplification was recovered in the progeny of the ADS6-containing strain.

Moreover, the hybridization signals of the amplified strains seemed to decrease in intensity at each round of sporulation. The relative hybridization signal intensities of the different clones in the lineage of NSA6 allowed us to conclude that the copy number of the amplification decreased even if we did not know the absolute copy number of the amplification. The loss of an amplification could thus be one of the multiple events occurring in the basic genetic instability. 


\section{AUD6 locus rearrangements in the stable Pig ${ }^{-}$mutants}

Spores of two $\mathrm{Pig}^{-}$(pigment-defective) colonies (PIG1 and PIG2), independently isolated in the progeny of two WT colonies, were plated on HT medium at the rate of about 100 spores per plate (five plates), incubated at $37^{\circ} \mathrm{C}$ and observed after growth for $5 \mathrm{~d}$. The progeny of these two Pig- colonies revealed only mutant clones exhibiting the parental mutant $\mathrm{Pig}^{-}$phenotype. These platings were classified as homogeneous stable progeny because no other phenotype was detected in the sample of colonies observed in the case of PIG1 and PIG2.

Genomic rearrangements at the AUD6 locus were investigated in a sample of 32 and 31 mutant colonies isolated from the progenies of PIG1 and PIG2, respectively. Hybridization patterns showed a very high degree of heterogeneity. Partial or complete deletions at the AUD locus were detected at high frequency (20/32 and $31 / 31$ for PIG1 and PIG2, respectively). Several rearrangements were more frequent than others. In the progeny of PIG1, one clone presented a $\Delta 2$ rearrangement, 15 a $\Delta 3$ one, 2 a $\Delta 5$ one and 2 a $\Delta 6$ one. In the progeny of PIG2, 11 clones showed a $\Lambda 3$ rearrangement, 5 a $\Delta 4$ one and 15 a $\Lambda 6$ one.

\section{Discussion}

\section{The AUD6 locus as a DNA rearrangement hotspot}

A high degree of genomic instability mainly affecting two chromosomal regions [AUD90 (P. Leblond, unpublished results) and AUD6] was revealed in mutant strains of $S$. ambofaciens DSM 40697 derived from independent genetic instability events. This work reports the rearrangements detected at the AUD6 locus. The structure found for the AUD6 family (12 AUDs in this family) is very similar to the AUD locus observed in S. glaucescens, in which heterogeneity in size and amplification degree was also observed (Häusler et al., 1989). According to the nomenclature proposed by Hütter et al. (1988), the AUD6 family of $S$. ambofaciens can be classified as type I amplifications.

DNA amplifications were previously reported in $21 \%$ of 'hypervariability-derived' strains (Leblond et al., 1989). Among 48 ADS-containing strains isolated from independent hypervariable lineages, $11(23 \%)$ were mapped in the AUD6 locus. Thus, a signal of amplification could exist in the AUD6 region. Deletions accompanying ADSs were found. These deletions occurred at one side of the ADSs and in two cases removed part of the proximal copy of the ADS. In addition, pedigree analyses of the amplified strain NSA6 revealed a loss of the amplification associated with a partial or complete deletion of the AUD6. The decrease of the relative hybridization signal intensities could be explained by the loss (partial or complete) of the ADS by deletion events. An alternative hypothesis could be a genomic heterogeneity of the different clones. These two processes could coexist: indeed, intramolecular recombination could result in changes of the apparent copy number as was reported by Jarman \& Wells (1989).

On the other hand, taking into account the three types of strain (A strains: phenotypically stable strains; B strains: unamplified hypervariable strains; $\mathrm{C}$ strains: ADS-containing strains), about $30 \%$ of the mutant strains presented rearrangements in the AUD6 region (16/30 A strains, 11/37 B strains and 0/29 C strains). Moreover, the deletions detected presented a different right endpoint in the various mutant strains studied. Twenty percent of the mutant strains studied harboured deletions ending in the AUD6, suggesting that this locus constitutes a rearrangement hotspot in $S$. ambofaciens DSM 40697. Indeed, these deletions presented an endpoint included in a $25 \mathrm{~kb}$ DNA region (AUD6) consisting of about $0.3 \%$ of the whole genome (estimated to about $8000 \mathrm{~kb}$ by Leblond et al., 1990 b). A signal of deletion endpoint (as an amplification signal) could thus exist in this region.

Therefore, the basic genetic instability is associated with a structural instability.

\section{The ability of the AUD6 locus to undergo sequential rearrangements}

Several observations suggested a multi-step process in the rearrangements of the AUD6 locus rather than a single-step mechanism. In the case of NSA6 and NSA 101 , a deletion event occurred after the amplification process. Thus, the loss of the amplification ADS6 also suggested a multi-step process. One mutant clone (NSA7) isolated from the lineage of the amplified strain NSA6 exhibited a deletion in the tandemly reiterated AUD6. These observations were consistent with a rearrangement within the AUD before the amplification process.

DNA pattern analyses of progenies of two pigmentnegative colonies revealed a high degree of genomic heterogeneity. A genomic hypervariability could be triggered during the development of a $\mathrm{Pig}^{-}$colony affecting the pigmentation genes: the genotypically heterogeneous colonies exhibited the $\mathrm{Pig}^{-}$phenotype. In one progeny, no WT AUD6 locus was recovered. On the basis of the existence of a single genome in a spore, the genomic hypervariability could result from several mutational events which occurred during the growth of 
the colony. This clonal effect suggests that these mutations could occur at different stages during colony development. Thus, the basic genetic instability could consist in a high frequency $(1 \%)$ of a primary mutational event inducing a genomic instability preferentially directed through specific loci such as the AUD6 locus.

\section{Genome plasticity in the AUD6 locus}

Leblond et al. (1990a) presented an hypothesis to explain the rearrangements in the upstream region of the amplifiable loci in the mutant strains. The principles of the model are applied here to the AUD6 locus to account for the high degree of plasticity of this locus in the phenotypically stable and hypervariable mutant clones.

In $S$. ambofaciens DSM 40697, P. Leblond and coworkers (unpublished results) have shown, using pulsedfield gel electrophoresis, that all the known rearrangements were located in one chromosomal arc. The extent of the deletions ranged between 250 and $2000 \mathrm{~kb}$. The two amplifiable regions were located in the chromosomal arc which showed the genome plasticity. In S. lividans, Cullum et al. (1988) have shown that the minimum size of the deletion associated with the amplification in the chloramphenicol-sensitive and $\mathrm{Arg}^{-}$mutants was of about $250 \mathrm{~kb}$. In $S$. glaucescens, the deletion sizes ranged from 250 to $800 \mathrm{~kb}$ (Birch et al., 1989).

The AUD6 contained a sequence homologous to several DNA fragments belonging to the AUD. An homologous AUD was found in another lineage of $S$. ambofaciens (ATCC 23877) and this AUD also presented these homologies. The AUD90 also showed such homologies but they were different from those of AUD6 (A. Dary, unpublished results). Moreover, the right endpoint of the AUD6 showed sequence homology with an internal sequence (IHS). A structure with repeated sequences was reported for the biosynthetic genes of erythromycin in Saccharopolyspora erythraea (Katz et al., 1990). S. ambofaciens produces a macrolide (spiramycin) but the restriction map of the AUD6 shows no similarity to the one published by Richardson et al. (1990). Nothing is known about the pigment produced by $S$. ambofaciens. By analogy with the results published by Davis \& Chater (1990), it could be a polyketide. The different deletions observed in the AUD6 could occur by recombination between two of these homologies. Thus, ADSs could be lost through two mechanisms. First, homologous recombination could occur between reiterated copies of the AUD. Secondly, recombination (homologous or illegitimate) events, analogous to those generating polar deletions in unamplified mutant strains and assumed to trigger amplifications (Leblond et al., 1990a), could occur between a sequence external to the ADS and a sequence belonging to the amplification.
This study was supported by grants from CNRS, from the Ministère de la Recherche et de l'Enseignement Supérieur, and from Université Nancy I. We thank the Société de Secours des Amis des Sciences and the Ligue Nationale Française Contre le Cancer for their financial support to one of us (D.S.).

\section{References}

Altenbuchner, J. \& Cullum, J. (1984). DNA amplification and an unstable arginine gene in Streptomyces lividans 66. Molecular and General Genetics 195, 134-138.

Altenbuchner, J. \& Cullum, J. (1985). Structure of an amplifiable DNA sequence in Streptomyces lividans 66. Molecular and General Genetics 201, 192--197.

Birch, A., Häusler, A., Vögtli, M., KreK, W. \& Hütter, R. (1989). Extremely large chromosomal deletions are intimately involved in genetic instability and genomic rearrangements in Streptomyces glaucescens. Molecular and General Genetics 217, 447-458.

BIRCH, A., HÄUSLER, A. \& HÜTTER, R. (1990). Genome rearrangement and genetic instability in Streptomyces spp. Journal of Bacteriology 172, 4138-4142.

Chater, K. F. \& Hopwood, D. A. (1984). Streptomyces genetics. In The Biology of the Actinomycetes, pp. 229-286. Edited by $M$. Goodfellow, M. Mordarski \& S. T. Williams. London: Academic Press.

Cullum, J., Altenbuchner, J., Flett, F., Piendl, W. \& Platt, J. (1986). DNA amplification and genetic instability in Streptomyces Biotechnology and Genetic Engineering Review 4, 59-78.

Cullum, J., Kaiser, P., Flett, F., Redenbach, M. \& Rauland, U. (1988). Genetic instability in Streptomyces lividans 66. In Abstracts of the 4th Conference on the Genetics and Molecular Biology of Industrial Microorganisms of the American Society for Microbiology, 1988. abstract no. 18 , p. 19.

Daniels, D. L., Schroeder, J. L., Blattner, F. R., Szybalski, W. \& SANGER, F. (1983). A molecular map of coliphage lambda. In: Lambda II, pp. 469-517. Edited by R. W. Hendrix, J. W. Roberts, F W. Stahl \& R. A. Weisberg. Cold Spring Harbor, NY : Cold Spring Harbor Laboratory.

DasGupta, U., Weston-Hafer, K. \& Berg, D. E. (1987). Local DNA sequence control of deletion formation in Escherichia coli plasmid pBR322. Genetics 115, 41-49.

DAvis, N. K. \& ChATER, K. F. (1990). Spore colour in Streptomyces coelicolor A3(2) involves the developmentally regulated synthesis of a compound biosynthetically related to polyketide antibiotics. Molecular Microbiology 4, 1679-1691.

Demuyter, P., Leblond, P., Decaris, B. \& Simonet, J. M. (1988). Characterization of two families of spontaneously amplifiable units of DNA in Streptomyces ambofaciens. Journal of General Microbiology' 134, 2001-2007.

FeinberG, A. P. \& Vogelstein, B. (1983). A technique for radiolabelling DNA restriction endonuclease fragments to high specific activity. Analytical Biochemistry 132, 6-13.

Hasegawa, M., Hintermann, G., Simonet, J. M., Crameri, R., Piret, J. \& Hütter, R. (1985). Certain chromosomal regions in Streptomyces glaucescens tend to carry amplifications and deletions. Molecular and General Genetics 200, 375-384.

Häusler, A., Birch, A., Krek, W., Piret, J. \& Hütter, R. (1989) Heterogeneous genomic amplification in Streptomyces glaucescens structure, location and junction sequence analysis. Molecular and General Genetics 217, 437-446.

Hopwood, D. A., Bibb, M. J., Chater, K. F., Kieser, T., Bruton, C. J., Kieser, H. M., Lydiate, D. J., Smith, C. P., Ward, J. M. \& SCHREMPF, H. (1985). Genetic Manipulation of Streptomyces - a Laboratory Manual. Norwich: John Innes Foundation.

HüTTER, R. (1967). Systematik der Streptomyceten. Basel: Karger Verlag.

HÜTTER, R. \& ECKHARDT, T. (1988). Genetic manipulation. In Actinomycetes in Biotechnology, pp. 89-184. Edited by M. Goodfellow, S. T. Williams \& M. Mordarski. London: Academic Press. 
Jarman, A. P. \& Wells, R. A. (1989). Hypervariable minisatellites: recombinators or innocent bystanders? Trends in Genetics 5, 367-371.

Katz, L., Staver, M., Tuan, J., Brown, D. \& Donadio, S. (1990). Genetics of erythromycin synthesis in Saccharopolyspora erythraea. In Abstracts of the UCLA Symposium of Molecular and Cellular Biology. (Journal of Cellular Biochemistry Supplement 14A, 1990), CC417, p. 129.

Leblond, P., Demuyter, P., Moutier, L., LaAkel, M., Decaris, B. \& SIMONET, J. M. (1989). Hypervariability, a new phenomenon of genetic instability, related to DNA amplification in Streptomyces ambofaciens. Journal of Bacteriology 171, 419-423.

Leblond, P., Demuyter, P., Simonet, J. M. \& DeCaris, B. (1990a). Genetic instability and hypervariability in Streptomyces ambofaciens: towards an understanding of a mechanism of genome plasticity. Molecular Microbiology 4, 707-714.

Leblond, P., Francou, F. X., Simonet, J. M. \& Decaris, B. (1990 $b$ ). Pulsed-field gel electrophoresis analysis of the genome of Streptomyces ambofaciens strains. FEMS Microbiology Letters 72, 79-88.

Maniatis, T., FritsCh, E. F. \& SAMBrook, J. (1982). Molecular Cloning, a Laboratory Manual. Cold Spring Harbor, NY: Cold Spring Harbor Laboratory.

Peeters, B. P. H., De Boer, J. H., Bron, S. \& Venema, G. (1988). Structural plasmid instability in Bacillus subtilis: effect of direct and inverted repeats. Molecular and General Genetics 212, 450-458.

Pridham, T. G., ANDERSon, P., Foley, C., Lindenfelser, L. A. \& BENEDICT, R. G. (1956/57). A selection of media for maintenance and taxonomic study of Streptomyces. Antibiotics Annuals 947-953.
Richardson, M. A., Kuhstoss, S., Huber, M. L. B., Ford, L., Godfrey, O., TURner, J. R. \& Nagaraja RaO, R.(1990). Cloning of spiramycin biosynthetic genes and their use in constructing Streptomyces ambofaciens mutants defective in spiramycin biosynthesis. Journal of Bacteriology 172, 3790-3798.

RoBINSON, M., LEWIS, E. \& NAPIER, E. (1981). Occurrence of reiterated DNA sequences in strains of Streptomyces produced by an interspecific protoplast fusion. Molecular and General Genetics 182 , 336-340.

SCHREMPF, H. (1983). Deletion and amplification of DNA sequences in melanin-negative variants of Streptomyces reticuli. Molecular and General Genetics 189, 501-505.

Short, J. M., Fernandez, J. M., Sorge, J. A. \& Huse, W. D. (1988) $\lambda$ Zap: a bacteriophage $\lambda$ expression vector with in vivo excision properties. Nucleic Acids Research 16, 7583.

SOUTHERN, E. M. (1975). Detection of specific sequences among DNA fragments separated by gel electrophoresis. Journal of Molecular Biology 98, 503-517.

Usdin, K., Christians, K. M., DeWet, C. A., Potgieter, T. D., SHAW, C. B. \& KIRBY, R. (1985). The loss of a large DNA fragment is associated with an aerial mycelium negative $\left(\mathrm{Amy}^{-}\right)$phenotype in Streptomyces cattleya. Journal of General Microbiology 131, 979-981.

Yanisch-PerRon, C., Vieira, J. \& Messing, J. (1985). Improved M13 phage cloning vectors and host strains: nucleotide sequences of the M13mp18 and pUC19 vectors. Gene 33, 103-119. 